\title{
Fax machines for thrombolysis?
}

The time dependence for successful reperfusion after thrombolytic treatment has motivated many strategies for reducing the delay between onset of symptoms and drug administration. Most of the delay is inevitably in the prehospital phase and thus difficult to influence. Even after hospital admission an hour or so may elapse before definitive treatment is started but this can more readily be addressed. Jargon phrases such as "door to needle time" and "time is muscle" were introduced to emphasise the need for rapid assessment and initiation of therapy. Simple checklists have been created to identify as rapidly as possible patients with contraindications to thrombolysis. ${ }^{2}$ In many centres, routine consultation with cardiologists has been discouraged because time is wasted finding an appropriate specialist physician, and subsequent second clinical assessments may add further delay.

Increasingly, therefore, the decision whether to administer thrombolytic agents has been left to relatively junior staff who are immediately available but who may not have had specialist training in cardiology. Centres administering thrombolytics in accident and emergency departments have been able to reduce average door to needle times for patients without contraindications to less than 30 minutes. ${ }^{3}$ The emphasis on speed has the potential for appreciable benefit to victims of acute myocardial infarction, but the possibility of forced errors must be considered when routine procedures are curtailed. New safeguards may be needed if old ones are abandoned.

The decision whether to administer thrombolytic drugs is not a trivial one in that reduction in mortality as a result of treatment may approach $50 \%$ if administration can be started within 90 minutes of the onset of symptoms, but it declines rapidly thereafter to a degree that little advantage may be achieved after 12 hours. ${ }^{4}$ Moreover, the benefits are partly offset by the risk of serious adverse effects-notably cerebral haemorrhage-that can be devastating to the $1 \%$ or so of patients who suffer them. Thus thrombolytic agents should not be administered in the absence of a firm indication lest harm be done to those who would not in any case have benefited; but neither should the drugs be withheld in doubtful cases if the opportunity for benefit is not overshadowed by the potential for harm. The responsibility for accurate assessment carried by relatively inexperienced junior medical staff is considerable. The question must be asked whether it is always appropriate for them to carry this burden.

Fortunately the indications and contraindications for thrombolytic therapy are usually clear, and simple checklists can provide firm guidance for the majority of cases. Difficulties arise, however, for three principal reasons. First, most contraindications to thrombolysis are only relative; the question of whether the potential gain is worth an identified increased risk of harm calls for mature judgment. Second, the time lapse since onset of symptoms may be difficult to define and the window of opportunity for benefit depends on factors that are complex and not well defined. Third, indications for treatment based on electrocardiographic criteria are not always straightforward. ST segment elevation may be evanescent and of doubtful significance, it may be a result of old infarction rather than representing any new event, or it may be physiological.

Therapeutic errors for such reasons will be familiar to all who care for patients with acute heart attacks. What should be done if the evidence for recent infarction is strong without
ST elevation in conventional electrocardiographic leads? Judgment may not be easy in the presence of bundle branch block or in the presence of other abnormal or unusual ECG patterns. These difficulties may well call for the opinion of a consultant or experienced registrar, but convenience and pragmatism often dictate otherwise.

Trainee medical staff must be encouraged to seek help for any decision that they find difficult and indeed for any that might be open to subsequent criticism. We fail them and our patients if support is not constantly available. Ideally any decision that relates to the appropriateness of thrombolysis should be made by clinicians who have seen the patient, but delays caused by a second tier of full clinical assessment may be appreciable and thus an expensive option in terms of lost time.

There is room for compromise. Advice can be given by telephone, but the electrocardiogram is also crucial to decisions that must balance benefit against risk. Fax transmission of 12-lead recordings is an option that was raised in the British Heart fournal in $1991 .^{5}$ We asked (rhetorically) how many cardiologists in district hospitals have fax machines in their homes both for this indication and as an aid to sound advice on the management of arrhythmias. We did not know the answer then and it will not be known now, but we believe the strategy deserves wider recognition. Srikanthan et al promote the concept in their paper in this issue, ${ }^{6}$ and emphasise its value out of working hours. In this study, 17 of 112 patients with suspected myocardial infarction had their management modified as a result of fax transmission of the electrocardiogram to a consultant who was at home: eight patients were saved unnecessary thrombolysis and four received it when otherwise they would not have done so. I suspect that similar figures would be found in other district hospitals that adopted the system. Fax transmission of electrocardiograms may be useful even in the daytime as senior medical staff are not always immediately available in hospitals with emergency units. Moreover, distances within a single institution and the many responsibilities that keep clinicians within one area constrain the possibilities for consultation.

Fax machines, which are relatively inexpensive in relation to hospital budgets for thrombolytic agents, have a role both in reducing door to needle times and in facilitating safer therapeutic decisions in relation to reperfusion therapy and arrhythmia management. Those who have responsibility for cardiac emergencies should recognise electrocardiographic transmission as a clinical tool. I believe that appropriate equipment should be readily available within the hospital and placed in the homes of cardiologists who have on call responsibilities for emergency care.

\section{CHAMBERLAIN} Hove, East Sussex, UK

1 MacCallum AG, Stafford PJ, Jones C, Vincent R, Perez-Avila C, Chamberlain DA. Reduction in hospital time to thrombolytic therapy by audit of policy guidelines. Eur Heart $\mathcal{F}$ 1990;11 (Suppl F):48-52.

2 More R, Moore K, Quinn E, Perez Avila C, Davidson C, Vincent R, et al. Delay times in the administration of thrombolytic therapy: the Brighton experience. Int $\mathcal{F}$ Cardiol 1995;49(Suppl):S39-46.

3 Birkhead JS. Time delays in provision of thrombolytic treatment in six district hospitals. BMF 1992;305:445-8.

4 Fibrinolytic Therapy Trialists (FTT) Collaborative Group. Indications for fibrinolytic therapy in suspected acute myocardial infarction; collaborative overview of early mortality and major morbidity results from all randomised trials of more than 1000 patients. Lancet 1994;343:311-22.

5 Chamberlain D, Oldershaw P. British Cardiac Society newsletter. Br Heart $\mathcal{f} 1991 ; 65: 298$.

6 Srikanthan VS, Pell ACH, Prasad N, Tait GW, Rae AP, Hogg KJ, et al. Use of fax facility improves decision making regarding thrombolysis in acute myocardial infarction. Heart 1997;78:198-200. 